\title{
Rheological Behavior of Al-Cu Alloys during Solidification: Constitutive Modeling, Experimental Identification, and Numerical Study
}

\author{
OLIVIER LUDWIG, JEAN-MARIE DREZET, CHRISTOPHE L. MARTIN, \\ and MICHEL SUÉRY
}

\begin{abstract}
The rheological behavior of a solidifying alloy is modeled by considering the deforming material as a viscoplastic porous medium saturated with liquid. Since the solid grains in the mush do not form a fully cohesive skeleton, an internal variable that represents the partial cohesion of this porous material is introduced. The model parameters are identified using shear and compressive stress states under isothermal conditions on an Al-Cu model alloy. The model is partially validated with nonisothermal conditions and we complete this study with tensile conditions. Such conditions, when applied on the mush, may lead to severe defects in many casting processes. The model has been implemented into a commercial finite-element code to simulate a tensile test. Comparison with experimental data shows that the model is able to reproduce the main features of a solidifying alloy under tension, although fracture is not directly addressed here. We show that two critical solid fractions must be introduced in the model to account for the rheology: the coherency solid fraction at which the mush acquires significant strength and the coalescence solid fraction at which solid grains start to form solid bridges.
\end{abstract}

\section{INTRODUCTION}

DURING solidification of metallic alloys, a continuous transition from liquid to solid state takes place. In the first stage of solidification, solid dendrites are completely free to move in the liquid matrix and the alloy can be considered, from a rheological point of view, as a suspension. As solidification progresses, solid fraction increases and interactions between dendrites begin to occur extensively. At this stage, the solid-liquid mixture is defined as a coherent mush (or "mushy zone") and develops shear strength due mostly to dendrite entanglement. ${ }^{[1,2,3]}$ The solid skeleton deforms essentially by rearrangement of dendrites at this stage and shear strength is of the order of tens of $\mathrm{kPa}^{[2,3]}$ For higher solid fractions, dendrites become highly interlocked and deformation of the mush must proceed both by rearrangement and deformation of the dendrites. The resulting shear strength is of the order of 0.1 to $1 \mathrm{MPa}$ and the mush also starts to develop tensile strength, although such strength is still very low. ${ }^{[2,3]}$ The solid fraction at which the mush develops such significant shear strength has been termed the "maximum packing solid fraction," $g_{s}^{p k}$, by Dahle and StJohn. ${ }^{[2]}$ At this point, the mush also exhibits some tensile strength that must be related to the establishment of mechanical coherence. Thus, arguably, we prefer to define this point as the coherency solid fraction $\left(g_{s}^{\text {coh }}\right)$. At this stage,

OLIVIER LUDWIG, formerly with the GPM2 Laboratory, UMR 5010, Institut National Polytechnique de Grenoble, is Postdoctoral Fellow, Computational Materials Laboratory, Ecole Polytechnique Fédérale de Lausanne, CH 1015 Lausanne, Switzerland. JEAN-MARIE DREZET, Senior Scientist, is with the Computational Materials Laboratory, Ecole Polytechnique Fédérale de Lausanne. CHRISTOPHE L. MARTIN, CNRS Research Associate, and MICHEL SUÉRY, CNRS Research Director, are with the GPM2 Laboratory, UMR 5010, Institut National Polytechnique de Grenoble, BP46 36402 St Martin d'Hères, Cedex, France. Contact e-mail: christophe.martin@inpg.fr

Manuscript submitted July 21, 2004. liquid fraction is still large enough to allow liquid feeding to accommodate local dilatation of the solid skeleton that constitutes the mush. Toward the very end of solidification, solid dendrites start to coalesce and solid permeability drops dramatically. Thus, the thin liquid films that remain in the mush at the end of solidification cannot accommodate tensile strains but still account for its fragile behavior. Any tensile strain at this stage is potentially dangerous for the integrity of the solidifying material. In particular, aluminum alloy processing technologies such as DC casting, laser welding, mold casting, or strip-casting involve thermally induced deformations arising from the contraction that occurs during casting (both solidification shrinkage and thermal contraction of the solid skeleton). These thermal strains can lead to the formation of casting defects such as macrosegregations (if liquid feeding is able to accommodate tensile strains) or porosity that may degenerate into hot tears.

In order to understand the formation of these defects, important modeling efforts have been carried out recently toward the development of thermomechanical models for the solidification of alloys ${ }^{[4,5,6]}$ and of hot tearing criteria. ${ }^{[7]}$ At some point, these models require constitutive equations addressing the rheological behavior of the mushy zone. In particular, the shear and tensile behaviors are believed to be of great importance for the initiation of the casting defects, although compressive stress states might also play a role in specific regions of the casting. The present rheological study is particularly directed toward the prediction of hot tearing in the aluminum DC casting process, where the accumulated strain in the mushy zone is relatively small (in any case less than $20 \mathrm{pct}$ ) and the strain rates are low (less than $10^{-3} \mathrm{~s}^{-1}$ ). The development of constitutive equations for such stress states requires suitable testing devices for obtaining experimental data.

In this article, we present a study of the rheological behavior of grain-refined $\mathrm{Al}-\mathrm{Cu}$ alloys. Constitutive equations of 
the mush are presented in a tensorial form, taking into consideration the main aspects of the mush that are relevant to the modeling of hot tears. ${ }^{[8]}$ The mushy zone is treated as a compressible viscoplastic porous medium saturated with liquid exhibiting some apparent strain hardening. ${ }^{[8,9]}$ The approach that has been adopted is presented in Section II, as well as the main equations that lead to the final constitutive model. The experimental identification of the model parameters and its validation are presented in Section III, where experimental data on solidifying alloys are given for various stress states, solid fractions, and strain rates. ${ }^{[10,11,12]}$ In Section IV, we present the work carried out on the tensile behavior of partially solidified alloys. This crucial stress state has been investigated experimentally and some numerical simulations of the tensile tests are required in order to exploit experimental data in an appropriate manner and to validate the model.

\section{CONSTITUTIVE MODEL FOR THE MUSHY ZONE}

In this section, we present the constitutive model that has been adopted to address the rheological behavior of the mushy zone. More details can be found in References 8 and 9. The solidifying alloy is treated as a viscoplastic porous medium saturated with liquid. Thus, the porous medium that constitutes the solid skeleton may densify under external pressure (expelling liquid) or dilate under tensile conditions (draining liquid). Furthermore, due to the discrete nature of the solidifying alloy made up of individual dendrites, the solid skeleton may not be fully cohesive. In other words, the thin liquid films that survive in the solidification process do not allow the mush to be considered as a fully cohesive porous material. ${ }^{[13]}$ The partial cohesion of the solidifying material is accounted for by introducing an internal variable $C$ that describes the state of cohesion of the mush. It is related to the amount of the total macroscopic strain that is transmitted at the scale of dendrites. ${ }^{[9]}$ The use of the internal variable $C$ allows the behavior of the solid skeleton to be described with two distinct limits, namely that of a cohesionless material for which all dendrites are fully wetted by the liquid $(C=0)$ and that of a fully cohesive porous material when dendrites have coalesced to such an extent that only isolated liquid pockets remain $(C=1)$.

\section{A. Model Framework}

The rheological model framework is such that it can be used continuously from the solid fraction limit at which significant shear and tensile strengths develop (coherency solid fraction $g_{s}^{c o h}$ ), up to the fully solid state. The constitutive equation is formulated on the solid skeleton and is written using the effective solid stress tensor $\hat{\sigma}_{s}$ that allows the effect of the liquid pressure $p_{l}$ on the solid phase to be taken into account via a hydrostatic term (neglecting liquid viscosity):

$$
\hat{\sigma}_{\mathrm{s}}=\sigma+p_{l} \mathbf{1}
$$

where $\sigma$ is the macroscopic stress tensor and $\mathbf{1}$ is the unit tensor. Such an approach is widely used in the field of soil mechanics, and has been already adopted by Martin et $a l . .^{[14,15,16]}$ and Zavaliangos ${ }^{[17]}$ to model the rheological behavior of partially remelted "semisolid" alloys. Once the effective solid stress tensor $\hat{\sigma}_{s}$ is introduced, it is possible to formulate the viscoplastic potential that characterizes the solid skeleton behavior (the elastic response is not detailed here; refer to Reference 18). First, we assume an associated flow rule:

$$
\dot{\varepsilon}_{s}^{p}=\frac{\partial \Omega}{\partial \hat{\sigma}_{\mathbf{s}}}
$$

that relates the solid phase plastic strain rate $\dot{\varepsilon}_{s}^{p}$ to the viscoplastic potential $\Omega\left(\hat{\sigma}_{\mathrm{s}}, g_{s}, T, C\right)$ via the volumetric solid fraction $g_{s}$, the temperature $T$, and the internal variable $C$. Neglecting the effect of the third invariant of the effective solid stress tensor, we write the viscoplastic potential expression as

$$
\Omega\left(\hat{\sigma}_{s}, g_{s}, T, C\right)=\Omega\left(\bar{P}_{s}, \bar{\sigma}_{s}, g_{s}, T, C\right)
$$

where $\bar{P}_{s}$ and $\bar{\sigma}_{s}$ are the effective pressure (taken positive in compression) on the solid skeleton and the von Mises stress, respectively:

$$
\begin{gathered}
\bar{P}_{s}=-\frac{1}{3} \operatorname{tr}\left(\hat{\sigma}_{s}\right) \\
\bar{\sigma}_{s}^{2}=\frac{3}{2} \mathbf{S}_{s}: \mathbf{S}_{s}
\end{gathered}
$$

with $\mathbf{S}_{s}$ denoting the solid phase deviatoric effective stress tensor $\left(\mathbf{S}_{s}=\hat{\sigma}_{s}-\frac{1}{3} \operatorname{tr}\left(\hat{\sigma}_{s}\right) \mathbf{1}\right)$.

\section{B. Viscoplastic Potential}

In a similar approach to the one adopted by Zavaliangos and Anand ${ }^{[18]}$ to describe the behavior of porous solid materials, we start by writing the viscoplastic potential $\Omega^{0}$ for a fully solid metallic alloy at high temperature. We use a simple power-law form:

$$
\Omega^{0}=\frac{\dot{\varepsilon}_{0} s_{0}}{n+1}\left(\frac{\bar{\sigma}_{s}}{s_{0}}\right)^{n+1}
$$

where $\dot{\varepsilon}_{0}=A \exp \left(-\frac{Q}{R T}\right)$ is the reference strain rate given by an Arrhenius expression. The parameters $A, Q, n$, and $s_{0}$ describe the rheology of the fully solid phase. In particular, $s_{0}$ has the dimension of a stress and represents an average isotropic resistance to plastic flow. Here, we assume that $s_{0}$ is constant, i.e., strain hardening effects are neglected in the solid phase. This assumption has been validated experimentally for fully solid Al-Cu alloys deformed at very high homologous temperature. ${ }^{[19]}$ Indeed, simple compression tests carried out in such conditions showed that strain hardening in the fully solid material is very limited. In the case of the fully solid material, the plastic strain rate tensor $\dot{\varepsilon}_{s}^{p}$ is thus simply

$$
\dot{\varepsilon}_{s}^{p}=\dot{\varepsilon}_{0}\left(\frac{\bar{\sigma}_{s}}{s_{0}}\right)^{n}\left\{\frac{3}{2}\left(\frac{\mathbf{S}_{s}}{\bar{\sigma}_{s}}\right)\right\}
$$

In order to write the viscoplastic potential for the mushy zone, a first step consists of taking into account the softening effect of liquid-saturated pores. In a similar fashion to what has been proposed in References 18, 20, and 21, 
we introduce a softening function $F$, which relates the viscoplastic potential $\Omega^{0}$ to the viscoplastic potential, $\Omega^{1}$, of the fully cohesive material with liquid-saturated pores $(C=1)$ :

$$
\Omega^{1}=\Omega^{0} F\left(\bar{P}_{s}, \bar{\sigma}_{s}, g_{s}, n\right)
$$

For the high solid fractions (low pore fractions) that are of interest here, we adopt a simplified form for $F$ :

$$
F\left(X, g_{s}, n\right)=\left(A_{2} X^{2}+A_{3}\right)^{\frac{n+1}{2}}
$$

where $X$ is the stress triaxiality $\left(X=\frac{\bar{P}_{s}}{\bar{\sigma}_{s}}\right)$ and $A_{2}$ and $A_{3}$ are solid fraction functions, which are given by Michel and Suquet: ${ }^{[20]}$

$A_{2}=\frac{9}{4}\left(n\left(\left(1-g_{s}\right)^{-1 / n}-1\right)\right)^{\frac{-2 n}{n+1}}, A_{3}=\left(1+\frac{2}{3}\left(1-g_{s}\right)\right)\left(g_{s}\right)^{\frac{-2 n}{n+1}}$

Equations [8] through [10] describe the behavior of the partially solidified alloy at the very end of solidification when liquid would still, hypothetically, be present as isolated pockets (Figure 1(a)). Earlier in the solidification process, this simplified picture is not correct. The presence of liquid films that are still present toward the end of solidification (Figure 1(b)) has some important consequences on the rheological behavior of the alloy: the solid network is not fully cohesive (strains are not totally transmitted through the solid skeleton) and the solid grains can rearrange themselves when submitted to a macroscopic strain. This is illustrated by Figure 2, which shows the comparison between the shear behavior of a partially solidified alloy and the behavior of a fully solid alloy, in similar conditions in terms of strain rate and temperature. For a fully solid alloy, 1 pct strain is sufficient for the stress to reach a steady-state value, whereas for a mushy zone submitted to a shear strain, 10 to 20 pct strain is necessary to reach a steady-state stress that is significantly lower than in the fully solid state, even at very high solid fraction.

We attribute this apparent "strain hardening" to the partial cohesion of the solid skeleton. In order to take this partial cohesion effect into account, the internal variable $C(0 \leq$ $C \leq 1$ ) has been introduced in the viscoplastic potential that describes the mush:

$$
\Omega=\Omega^{0} \frac{F}{C^{n}}
$$

$C=1$ corresponds to a fully cohesive porous material (Eq. [8]) whereas $C \rightarrow 0$ corresponds to a material for which

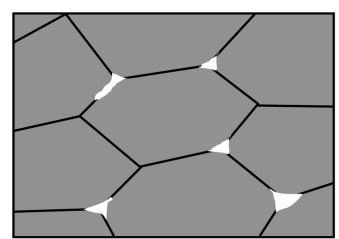

(a)

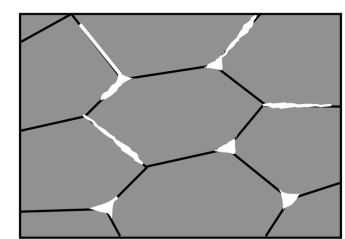

(b)

Fig. 1-Schematic of the structure of (a) a cohesive porous solid alloy saturated with liquid and $(b)$ a partially cohesive porous solid alloy. all grains are wetted by liquid. Replacing $F$ by its expression in Eq. [8], the viscoplastic potential $\Omega$ becomes:

$$
\Omega=\frac{\dot{\varepsilon}_{0}}{(n+1)\left(C s_{0}\right)^{n}}\left(A_{2} \bar{P}_{s}^{2}+A_{3} \bar{\sigma}_{s}^{2}\right)^{\frac{n+1}{2}}
$$

Note that $C s_{0}$ may be interpreted from Eq. [12] as the average isotropic resistance to plastic flow that is transmitted to the grains. The plastic strain rate tensor of the solid phase for the partially cohesive mush is derived from the normality rule (Eq. [2]):

$$
\dot{\varepsilon}_{s}^{p}=\frac{\dot{\varepsilon}_{0}}{\left(C s_{0}\right)^{n}}\left\{-\frac{A_{2}}{3} \bar{P}_{s} \mathbf{1}+\frac{3}{2} A_{3} \mathbf{S}_{s}\right\}\left\{A_{2} \bar{P}_{s}^{2}+A_{3} \bar{\sigma}_{s}^{2}\right\}^{\frac{n-1}{2}}
$$

Equation [13] is the constitutive equation of the mush. It shows that the behavior of the solid skeleton that constitutes the mush is pressure sensitive whenever solid fraction is less than unity $\left(A_{2}>0\right)$. Also, due to the even form of $F$ relative to $X$ (Eq. [9]), the behavior of the mush is symmetric for tensile $(X<0)$ and compressive $(X>0)$ conditions for a given value of the cohesion variable $C$. Experimentally, it has been shown that there exists a strong dissymmetry between tensile and compressive stress states. Partially solidified alloys exhibit a much weaker response in tension compared to compression. ${ }^{[16]}$ This dissymmetry is rendered in the model through the internal variable $C$ that evolves differently in tensile and compressive conditions. The advantage of the present formulation is that in the context of an associated flow potential (Eq. [2]), the dilatation rates given by the model in tensile conditions are in agreement with the limited experimental data available to us. ${ }^{[16]}$

\section{Evolution Equation for the Internal Variable $C$}

The internal variable $C$ that accounts for the partial cohesion of the mushy zone is assumed to evolve with time

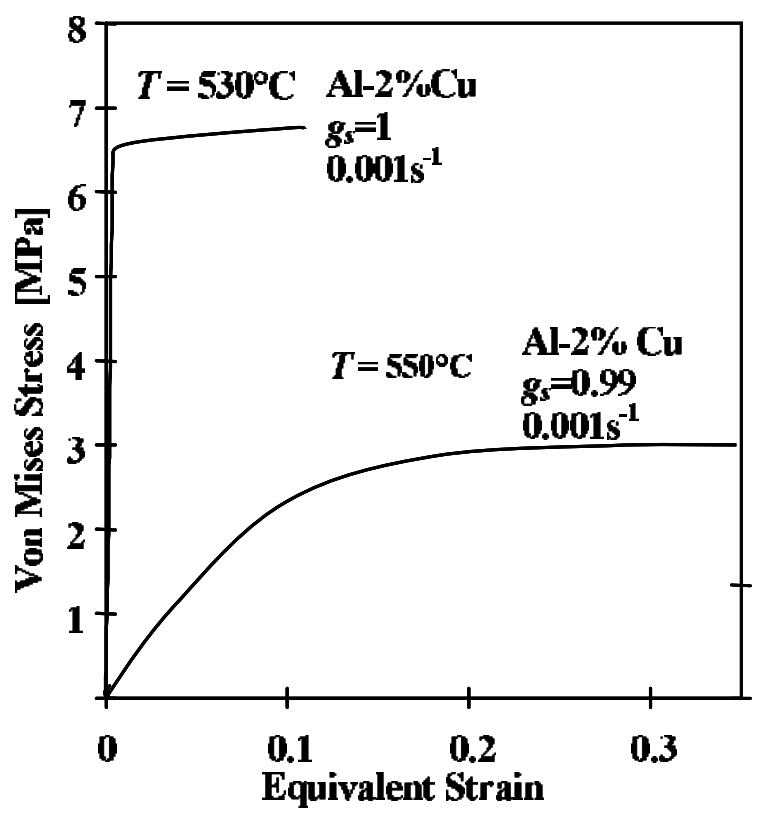

Fig. 2-Von Mises stress $v s$ strain curves for an Al-2 wt pct $\mathrm{Cu}$ alloy, tested in simple compression in the fully solid state and in shear condition in the mushy state (experimental results in Section III-A), for the same strain rate and at a similar temperature. 
owing to the rearrangement of solid grains. The various alternatives for writing the evolution equation of $C$ have already been discussed in the case of pure shear in Reference 9. The approach consists of considering that $C$ increases via the formation of contacts between solid grains and decreases by rearrangement of grains or contact failure. Here, we simply assume that both the increase and the decrease of $C$ scale with a measure of the macroscopic strain rate $\dot{\varepsilon}_{e}$. Therefore, we write the evolution equation of the internal variable in the form

$$
\frac{\mathrm{d} C}{\mathrm{~d} t}=\alpha\left(g_{s}, X\right)\left(1-\frac{C}{C^{*}\left(g_{S}, X\right)}\right) \dot{\varepsilon}_{e}
$$

where $\dot{\varepsilon}_{e}=\sqrt{\frac{2}{3} \dot{\varepsilon}_{s}^{p}: \dot{\varepsilon}_{s}^{p}}$ is a scalar measure of the macroscopic plastic strain rate of the solid skeleton. Equation [14] carries some similarity with other evolution equations that have been proposed in the literature for characterizing a semisolid or a partially solidified alloy with an internal variable that accounts for the agglomeration between solid grains or the cohesion of the mush. ${ }^{[17,22]}$ The rheological functions $\alpha\left(g_{s}, X\right)$ and $C^{*}\left(g_{s}, X\right)$ govern the evolution of $C$ with macroscopic strain and depend both on the solid fraction and on the stress state (via the stress triaxiality $X$ ). The function $\alpha\left(g_{s}, X\right)$ governs the evolution of $C$ at small strains, whereas $C^{*}\left(g_{s}, X\right)$ defines the saturation value of $C$ at large strains.

The flow Eq. [13] together with the evolution Eq. [14] address the rheological behavior of the partially solidified alloys in the entire stress space (the third invariant effect is neglected, as mentioned previously) and for any solid fraction above which the mush develops significant shear and tensile strength $\left(g_{s}>g_{s}^{c o h}\right)$. The next step consists of determining suitable expressions for the rheological functions $\alpha\left(g_{s}, X\right)$ and $C^{*}\left(g_{s}, X\right)$. Therefore, experimental identification of these functions has to be carried out by means of mechanical testing of partially solidified alloys in different stress states, for different strain rates and solid fractions.

\section{EXPERIMENTAL IDENTIFICATION AND VALIDATION IN SHEAR AND DRAINED COMPRESSION}

The experimental investigation of the behavior of partially solidified alloys is the scope of parts III and IV. The tested materials are grain-refined $\mathrm{Al}-\mathrm{Cu}$ alloys obtained from aluminum 1085 (99.88 wt pct) and pure copper. The alloy is inoculated with an AT5B master alloy (Al-5 wt pct Ti-1 wt pct B) during the casting using $5 \mathrm{~kg}$ of AT5B per ton. The typical size of the grains after solidification with the cooling rates used here is of the order of $200 \mu \mathrm{m}$. The grain refiner allows an equiaxed microstructure to be obtained. This enables us to assume that the mechanical properties of a volume element of the mushy zone are isotropic. In References 10 and 11, we have used a simple Scheil equation to compute the solid fraction from the test temperature. Here we use a numerical solution that takes back diffusion of copper into account during the solidification path, using a phase formation model described in Reference 23. In that case, we obtain slightly higher solid fractions compared to the Scheil equation.
In the tests described hereafter, the time required to stabilize the temperature is 200 seconds. Such a holding time has been minimized, in order to reduce microstructural changes. The effect of isothermal holding on the microstructure of a partially solidified $\mathrm{Al}-\mathrm{Cu}$ alloy has been studied in detail by Braccini et al. ${ }^{[24]}$ The results showed that the morphological evolution was much slower when the alloy was grain refined and that it should be a second-order effect for the durations that are involved in our experimental conditions.

\section{A. Pure Shear}

The shear behavior of a grain-refined $\mathrm{Al}-2$ wt pct $\mathrm{Cu}$ alloy was investigated by means of a specially designed translation shear test device detailed in Reference 10 . The test consists of deforming a solidifying alloy located between two coaxial cylinders (Figure 3). The alloy is initially totally melted and is then partially solidified at a controlled constant cooling rate. At a given temperature, a constant velocity is imposed to the inner cylinder, thus shearing the alloy at a constant rate. Two types of tests have been performed: an isothermal test and a nonisothermal one during which the temperature is decreased at a given rate.

Isothermal shear tests allow the effects of strain, strain rate, and solid fraction to be investigated in a decoupled manner. Typical results are shown in Figure 4, where the measured von Mises stress (thick line curves) is plotted against the imposed macroscopic strain. Qualitatively, all curves exhibit an apparent increase of stress with strain before reaching a steady-state value after 0.1 to 0.2 strain. This shows that the isothermal shear behavior is particularly sensitive to the accumulated strain experienced by the mushy zone. This apparent strain hardening at small strains is accounted for in our model, via the evolution of $C$ (Eq. [14]). Concerning the steady-state stress at large strains, Figure 4 shows that it increases with increasing solid fraction and applied strain rate as observed classically for semisolid alloys and partially solidified alloys. ${ }_{1}^{[14,15]}$ The strain rate sensitivity of the steady-state stress, $\frac{1}{n}$, has been calculated from this series of tests, and the value of $n(n=3.8)$ may be considered as characteristic of the fully solid phase behavior.

\section{Imposed displacement}

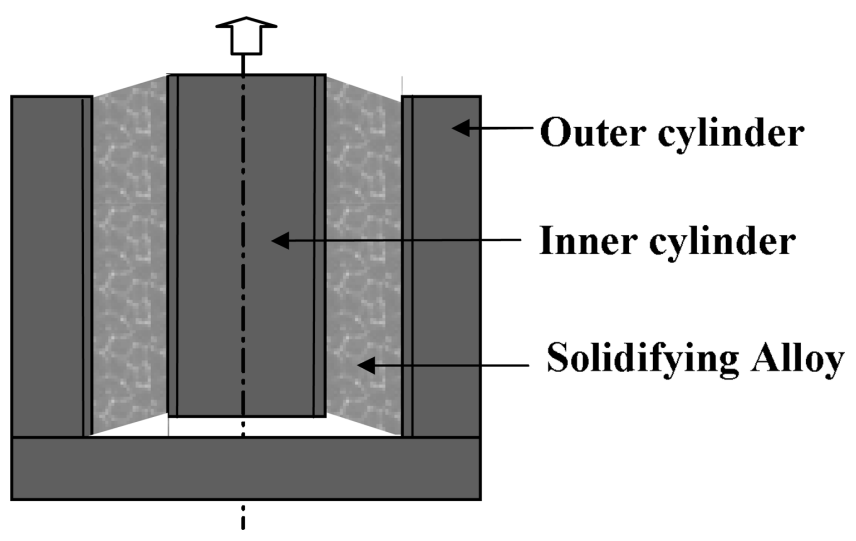

Fig. 3-Schematic of the translation shear test apparatus. 


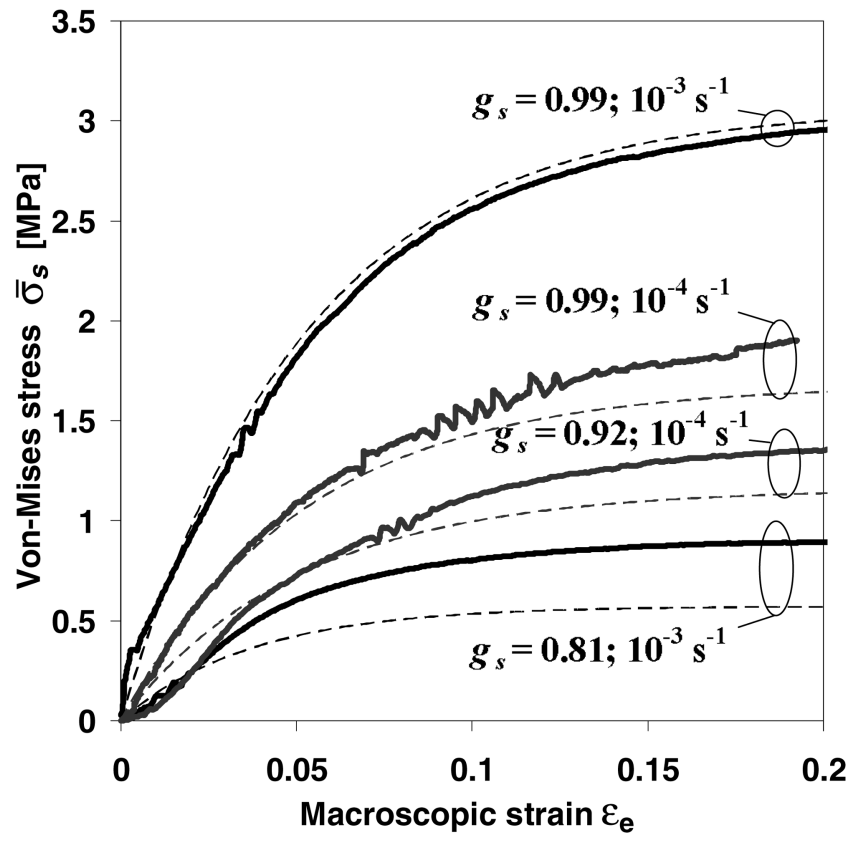

Fig. 4-Evolution of the von Mises stress with equivalent strain for various strain rates and solid fractions in pure shear. Experimental data (continuous curves) and model (dotted curves) for an $\mathrm{Al}-2$ pct wt $\mathrm{Cu}$ at $g_{s}=0.81,0.92$, 0.99 corresponding to $T=635^{\circ} \mathrm{C}, 610^{\circ} \mathrm{C}$, and $550{ }^{\circ} \mathrm{C}$, respectively.

The data of isothermal shear tests were used to identify the rheological functions in pure shear $\left(X=\bar{P}_{S}=0\right)$. For this stress state, Eq. [13] reduces to a simple scalar equation. Assuming that the solid fraction remains constant during an isothermal test, the evolution of $C$ is calculated by analytical integration of Eq. [14]. By fitting the stress vs strain curves and assuming that the rheological functions are strainrate independent, we have determined their expressions for $X=0$ :

$$
\begin{aligned}
& \alpha\left(g_{s}, X=0\right)=\alpha_{0}+\alpha_{1} \frac{g_{s}^{\frac{1}{3}}}{1-g_{s}^{\frac{1}{3}}} \\
& C^{*}\left(g_{s}, X=0\right)=1-\left(1-g_{s}\right)^{p}
\end{aligned}
$$

where $\alpha_{0}=4.45, \alpha_{1}=1.07 \cdot 10^{-2}$, and $p=0.11$ are fitted parameters. These expressions were chosen for their simplicity, and we have verified that strain-rate-independent functions give satisfactory results compared to the experimental data. This last point was studied in more detail in Reference 9 for a $\mathrm{Sn}-\mathrm{Pb}$ model alloy and in Reference 19 for the present system. Here, we state that the rheological functions $\alpha$ and $C^{*}$ may be considered strain rate independent in the limited strain rate domain where the functions have been fitted $\left(10^{-4}\right.$ to $\left.10^{-3} \mathrm{~s}^{-1}\right)$. Also, when the solid fraction reaches unity, a purely viscoplastic behavior, characteristic of a fully solid alloy at high temperature, is obtained $\left(C^{*}=1, \alpha \rightarrow \infty\right)$. The dotted lines in Figure 4 show the calculated stress-strain behavior for each corresponding experimental condition using these functions. The agreement with experimental data is fair, considering the simplicity of Eqs. [15] and [16].

The nonisothermal shear test allows the behavior of the solidifying alloys to be investigated in more realistic and

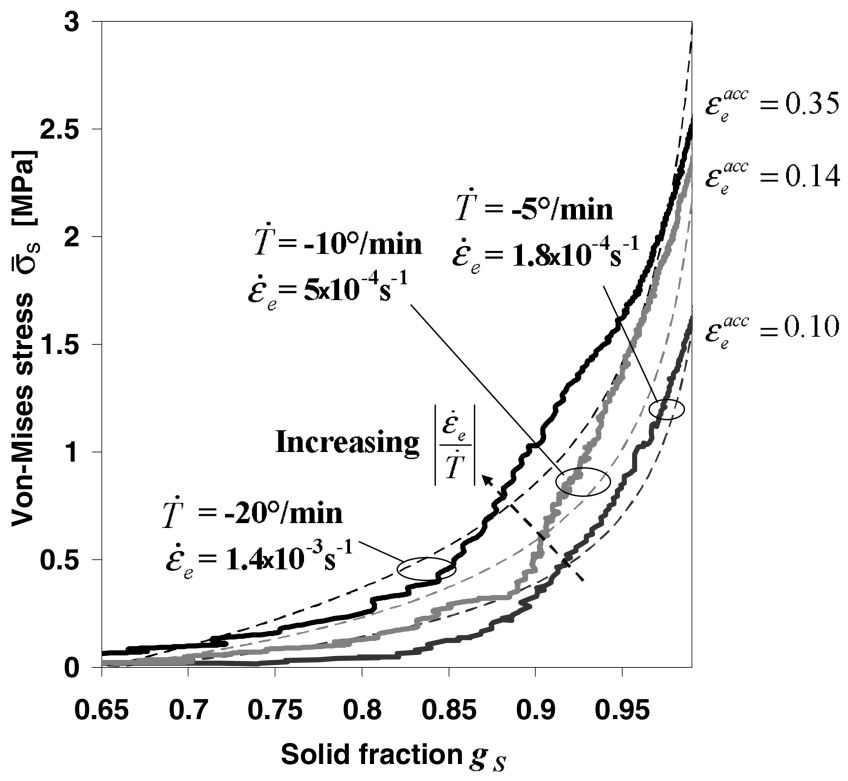

Fig. 5-Nonisothermal shear tests: comparison between experimental results (continuous curves) and model prediction (dotted curves) for various experimental conditions in terms of strain rate and cooling rate.

more complex conditions. During this test, the alloy is strained while the solid fraction is increasing. In Figure 5, the results are plotted in terms of the von Mises stress vs solid fraction. The macroscopic strain $\varepsilon_{e}^{a c c}$ that the alloy has accumulated during solidification is also indicated to show that for a given solid fraction, the behavior is strain dependent. Note that the accumulated strain is directly proportional to the ratio between the imposed macroscopic strain rate $\dot{\varepsilon}_{e}$ and the cooling rate $\dot{T}$.

These results are used to validate the present rheological model in pure shear. Equation [14] has been integrated numerically for nonisothermal conditions, using the rheological functions given by Eqs. [15] and [16] (determined in isothermal conditions). The evolution of the von Mises stress with the solid fraction is then calculated using Eq. [13] for $X=\bar{P}_{S}=0$.

From Figure 5, we can conclude that the agreement between the experimental and calculated curves is satisfactory. In particular, Figure 5 shows that the model reproduces correctly the behavior of the mush for various values of the $\dot{\varepsilon}_{e} / \dot{T}$ ratio. Figure 5 suggests that in practical conditions, it is the $\dot{\varepsilon}_{e} / \dot{T}$ ratio that dictates the stress buildup in the mush. This ratio is determined by the process conditions in DC casting. Thus, we believe that the present model, which has been identified in isothermal conditions, is able to predict correctly the shear behavior of the mushy zone in solidification conditions that are close to those encountered in real DC casting.

\section{B. Drained Compression Behavior}

In order to study the behavior of the mushy zone in a stress state where densification of the solid skeleton operates $(X>0)$, a drained compression apparatus has been designed (Figure 6 for a schematic and Reference 12 for more details on the experimental procedure). During the test, the alloy is 


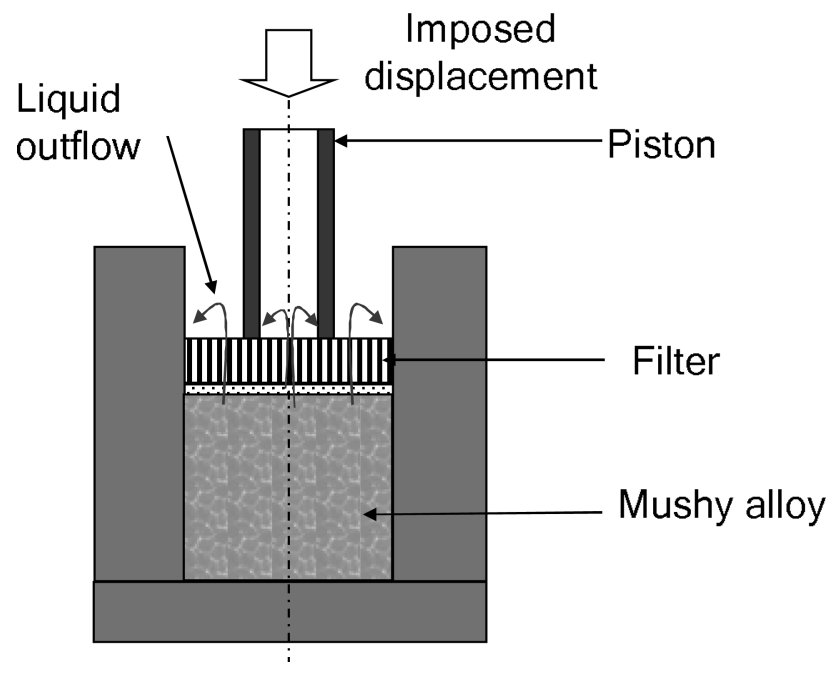

Fig. 6-Experimental setup for drained compression tests.

initially melted, and then partially solidified until a given solid fraction $g_{s}^{0}$ is reached. At this stage, the temperature is kept constant, and a downward vertical displacement is imposed to the hollow piston. A filter allows the liquid to flow out of the sample, and, consequently, the solid fraction increases. This test may be interpreted as a means to impose solidification mechanically at constant temperature. Optical micrographs of the tested sample revealed that liquid only is expelled through the filter and that the densification of the solid skeleton under the filter may be considered as homogeneous. Assuming that the solid fraction evolves only by liquid drainage, one can relate the imposed axial strain $\varepsilon_{z}$ to the solid fraction:

$$
g_{s}=g_{s}^{0} \exp \left(-\varepsilon_{z}\right)
$$

where $g_{s}^{0}$ is the initial solid fraction before any strain is imposed. Several tests have been carried out for various initial solid fractions $(0.62,0.70$, and 0.84$)$ by varying the copper content of the alloy at the same temperature $(15,12$, and 8 wt pct $\mathrm{Cu}$, respectively). The results are exploited by plotting the measured axial stress as a function of the solid fraction $g_{s}$. Such results are shown in Figure 7 and reveal that the stress increases with increasing solid fraction, and that the value of $g_{s}^{0}$ has an effect on the measured stress, up to a solid fraction of about 0.9. For higher solid fractions, the different curves merge and the axial stress tends to very large values. When the solid fraction is close to unity, the densification of the solid skeleton is almost complete, and the stress increases asymptotically, owing to the incompressibility of the (almost) fully solid alloy.

When subjected to compressive stress states like those involved in this test, the solid dendrites are likely to form contacts, thereby increasing the cohesion of the solid skeleton. It is therefore reasonable to state that the saturation value of $C$ will be much higher than its value in pure shear or in dilatation stress states. However, we have very little information about the deformation mechanisms responsible for the initial increase of $C$. For these reasons, and for the sake of simplicity, we make the following hypothesis on the rheological functions for the stress states involved in the drained compression test:

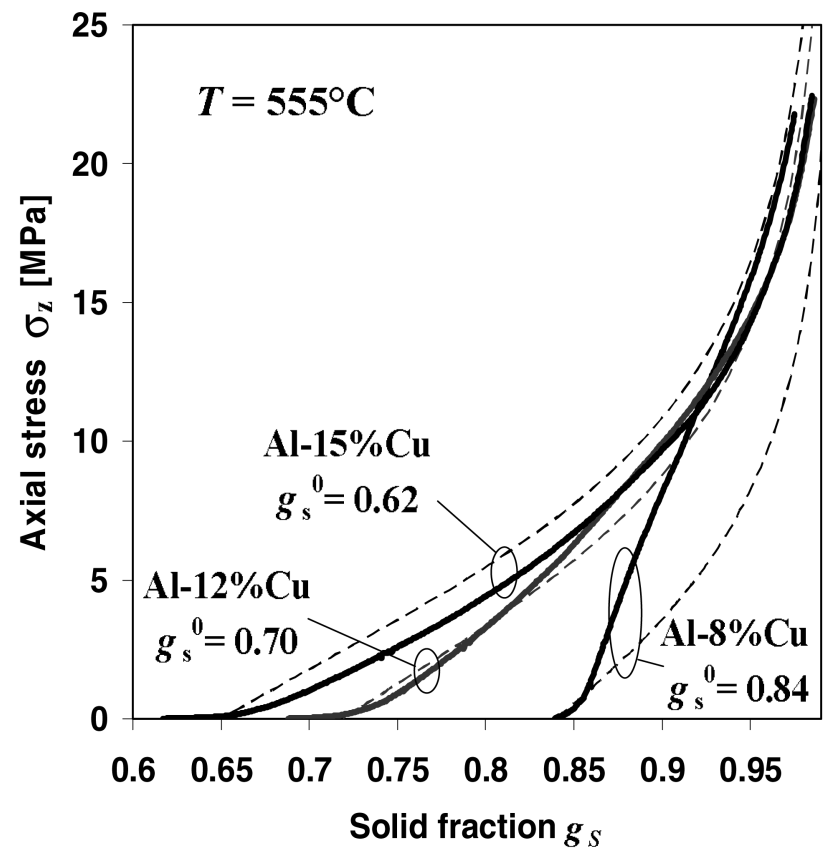

Fig. 7-Drained compression behavior of Al-Cu alloys: comparison between experimental results (continuous curves) and model prediction (dotted curves) under the hypothesis of negligible liquid pressure.

$$
\begin{gathered}
C^{*}\left(g_{s}, X>2\right)=1 \\
\alpha\left(g_{S}, X>2\right)=\alpha\left(g_{S}, X=0\right)=\alpha_{0}+\alpha_{1} \frac{g_{S}^{\frac{1}{3}}}{1-g_{S}{ }^{\frac{1}{3}}}[19]
\end{gathered}
$$

The choice of setting $C^{*}$ to unity for $X>2\left(\bar{P}_{s}>2 \bar{\sigma}_{s}\right)$ is somewhat arbitrary and is linked to the paucity of our experimental data for stress states in between pure shear and drained compression. Only triaxial tests that consist of applying axial strain while a controlled lateral pressure is imposed on the specimen would give enough information to propose a more realistic choice. Such tests that are routinely used in soil mechanics (for example, Reference 25 for viscoplastic materials) are difficult for solidifying alloys with low melting point ${ }^{[15]}$ and are clearly out of the reach for partially solidified aluminum alloys. Note that, according to the model, the drained test is characterized in the stress space by a triaxiality that is larger than two whatever the solid fraction. Furthermore, we assume that the interstitial pressure of the liquid is negligible as compared to the stresses necessary to densify the solid skeleton. This assumption seems reasonable at the beginning of the densification process when the permeability of the liquid-saturated solid skeleton is still significant. Under these simplifications, Eqs. [13] and [14] provide the axial stress as a function of the solid fraction.

Figure 7 shows that the model (dotted curves) reproduces correctly the general trends given by the experimental curves (continuous curves). The agreement is reasonable except for the highest initial solid fraction $\left(g_{s}^{0}=0.84\right)$. This discrepancy can be attributed to the effect of the liquid pressure, which has been neglected here. Similar computed curves were obtained by numerical simulations of the drained compression test, using $\mathrm{ABAQUS}$ and assuming a negligible liquid pressure. ${ }^{[12]}$ 


\section{TENSILE BEHAVIOR}

In the various theories proposed in the literature to model hot tearing, it is generally admitted that tensile strains are of crucial importance in the formation of this casting defect. ${ }^{[7,26-32]}$ Therefore, the tensile behavior of partially solidified alloys has been the focus of numerous studies (in particular, References 27 and 32 provide a review). In the present work, we investigate this stress state that can lead to fracture. We do not intend to propose a fracture criterion per se, but to show that the rheological model that has been described in Section II and identified and partially validated in Section III is able to reproduce the main features of the stress-strain response observed before fracture.

\section{A. Tensile Tests}

The experimental tensile setup used in this study is illustrated in Figure 8 and has been detailed in Reference 33. It consists of a vertical tensile machine equipped with watercooled grips and induction heating. The temperature is measured with a thermocouple located close to the center of the specimen. It has been verified that the thermocouple does not have a significant mechanical effect. ${ }^{[19]}$ The initially solid specimen is completely melted by induction heating in its middle part. It is then cooled at a controlled cooling rate of $1{ }^{\circ} \mathrm{C} \mathrm{s}^{-1}$ until a given temperature is reached in the solidification range at the location of the thermocouple. This temperature is stabilized for 90 seconds, and deformation is then carried out at a constant displacement rate. The difficulty concerning this test, compared to the shear and drained compression tests described in preceding sections, is that temperature is not homogeneous in the specimen. The zone that was partially solidified during the test is well characterized (it is 20 - $\mathrm{mm}$ high, and $9 \mathrm{~mm}$ in diameter), but there exists a solid fraction gradient along the tensile axis in this zone.

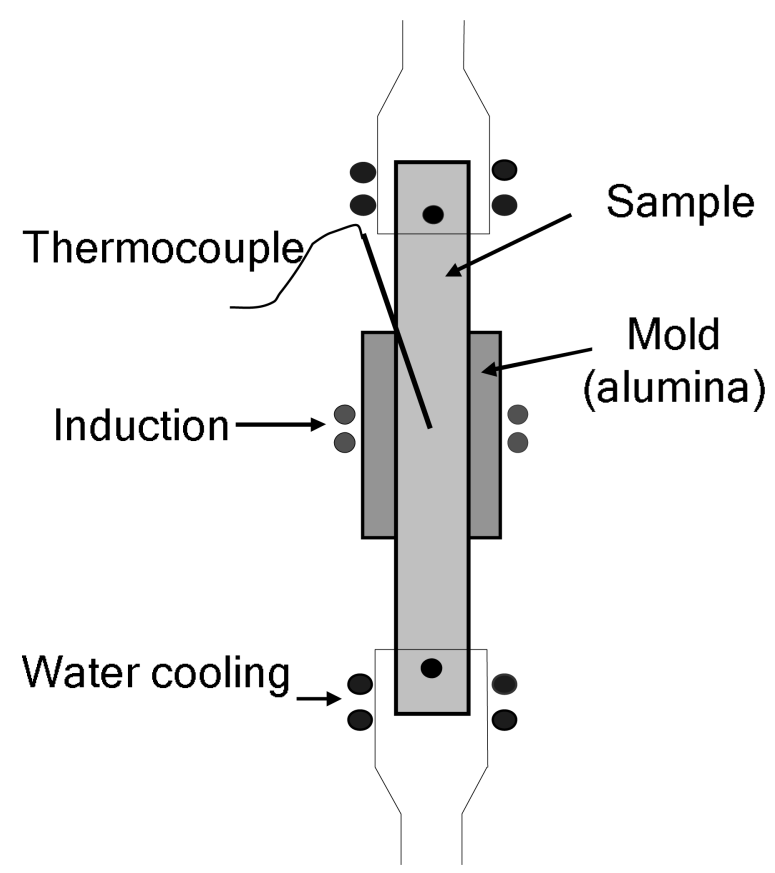

Fig. 8-Schematic of the tensile experimental setup.
This leads to a heterogeneous strain field in the deformation zone along the tensile axis. Note that we have verified that radial temperature gradients are minimal for our test conditions.

In this context, we prefer to present the tensile data as axial stress $v s$ axial displacement, instead of axial stress $v s$ a hypothetic axial strain. Figure 9 shows typical curves from the tensile test. The stress increases with increasing displacement and reaches a maximum value $\sigma_{\max }$ before fracture of the specimen occurs. Two different behaviors are observed depending on the solid fraction investigated (the solid fraction investigated refers to the lowest solid fraction in the specimen at the center of the specimen). Below 0.94 solid fraction, we observe a gradual increase of the axial stress followed by a gradual decrease down to the final fracture of the material. At larger solid fractions $\left(g_{s}>0.94\right)$, the stress increase is sharper, and is followed by a sudden drop.

The maximum tensile stress (that we denote as the tensile strength) is plotted against the solid fraction at the center of the sample in Figure 10. The tensile strength increases with increasing tested solid fraction. In accordance with Figure 9. Figure 10 shows that the maximum tensile stress increases sharply for solid fraction larger than 0.94 . Thus, we believe that there exists a significant microstructural modification occurring around this solid fraction. All tensile tests have been carried out at temperatures equal to or greater than $550{ }^{\circ} \mathrm{C}$. For an $\mathrm{Al}-\mathrm{Cu}$ alloy, the equilibrium eutectic temperature is $548{ }^{\circ} \mathrm{C}$. Moreover, our numerical calculations, which take back diffusion of copper into account during the solidification path, indicate an undercooling of a few degrees for the cooling rates that we have used. Therefore, we believe that during the tensile tests, no eutectic was solidified in the central part of the sample.

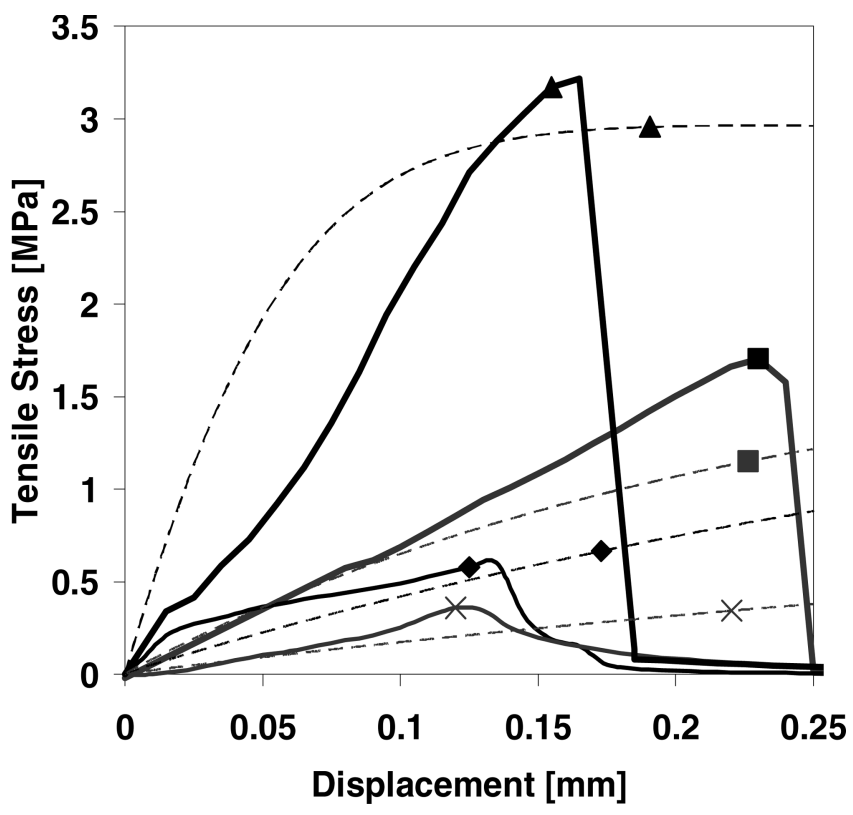

Fig. 9-Tensile behavior of partially solidified Al-Cu alloys: stress vs displacement curves from experimental data (continuous curves) and from the model (dotted curves) for various solid fractions. $\times g_{s}=0.92, T=610^{\circ} \mathrm{C}$ ( 2 wt pct $\mathrm{Cu}$ ); $g_{s}=0.94, T=551{ }^{\circ} \mathrm{C}\left(4\right.$ wt pct $\mathrm{Cu}$ ); $g_{s}=0.96, T=$ $585^{\circ} \mathrm{C}(2$ wt pet $\mathrm{Cu}) ; \boldsymbol{\Delta} g_{s}=0.98, T=551{ }^{\circ} \mathrm{C}(2$ wt pet $\mathrm{Cu})$. 


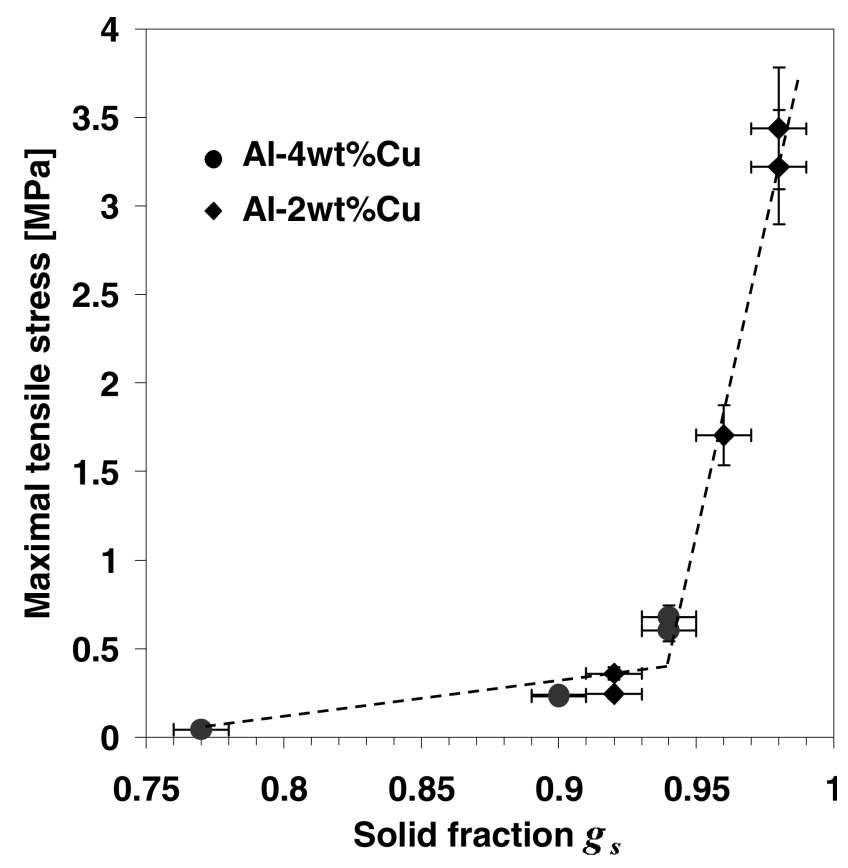

Fig. 10-Maximal tensile stress vs solid fraction for two different compositions.

Rappaz et al. ${ }^{[34,35]}$ have shown that coalescence starts to operate at solid fractions comprised between 0.93 and 0.96 . Thus, we associate the sharp increase in strength to the formation of solid bridges between dendrites brought by coalescence. Rappaz and co-workers have shown that coalescence depends on interfacial energies as well as on the morphology of solid grains. ${ }^{[13,34,35]}$ Also, the presence of very thin liquid films (a few nanometers, according to these authors) at the very end of solidification renders the determination of the existence of solid bridges difficult by classical microstructural characterization techniques. However, fracture surface observations of tensile specimens in relation with strength measurements can give relevant qualitative information concerning the state of coalescence. The observed fracture surfaces present a dendritic morphology with (eutectic) liquid films covering the dendrites arms. For solid fractions above 0.94 , some signs of the existence of solid bridges were clearly detected, as shown by the scanning electron microscopy observation in Figure 11. We denote $g_{s}^{\text {coal }}$ as the critical solid fraction, and we evaluate $g_{s}^{\text {coal }}=0.94$ for the grain-refined Al-Cu alloys investigated here (note that compositions with 2 wt pct and 4 wt pct $\mathrm{Cu}$ have been tested without observing a detectable influence of the copper content on $g_{s}^{\text {coal }}$ ).

\section{B. Finite-Element Simulation}

As mentioned in Section A, there exists an axial solid fraction gradient for the tensile test. This gradient does not allow a simple analytical solution to be used for comparing the model prediction and the experimental data in tensile conditions. Thus, in order to test the validity of the model under strain conditions that lead to hot tearing, numerical simulations of the test have been carried out using the finite-element software ABAQUS 6.3. As shown in Figure 12 , only one quarter of the specimen was modeled and

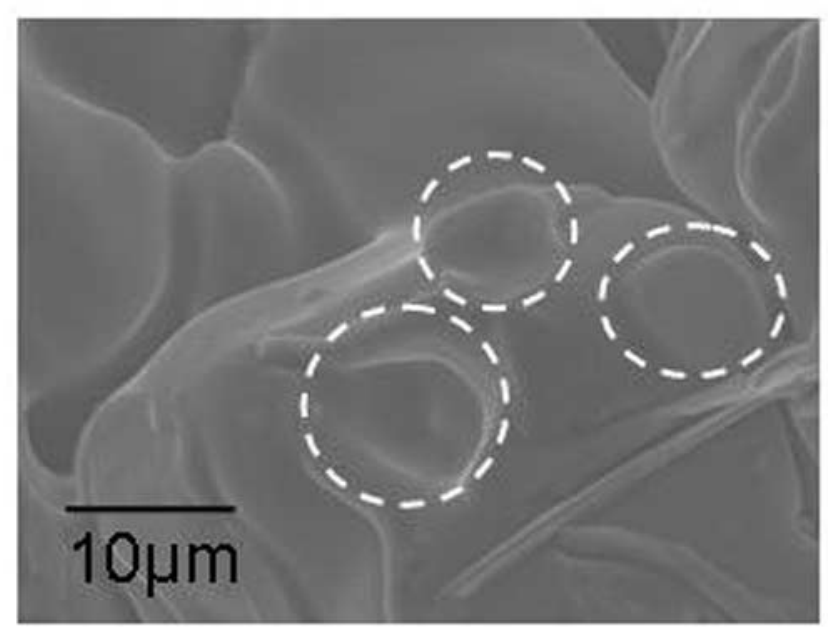

Fig. 11-Scanning electron microscopy (secondary electrons) of the fracture surface of a tensile specimen tested at a solid fraction of 0.96 , showing evidence of ductile fracture of solid bridges on dendrites arms (white dotted ellipses).

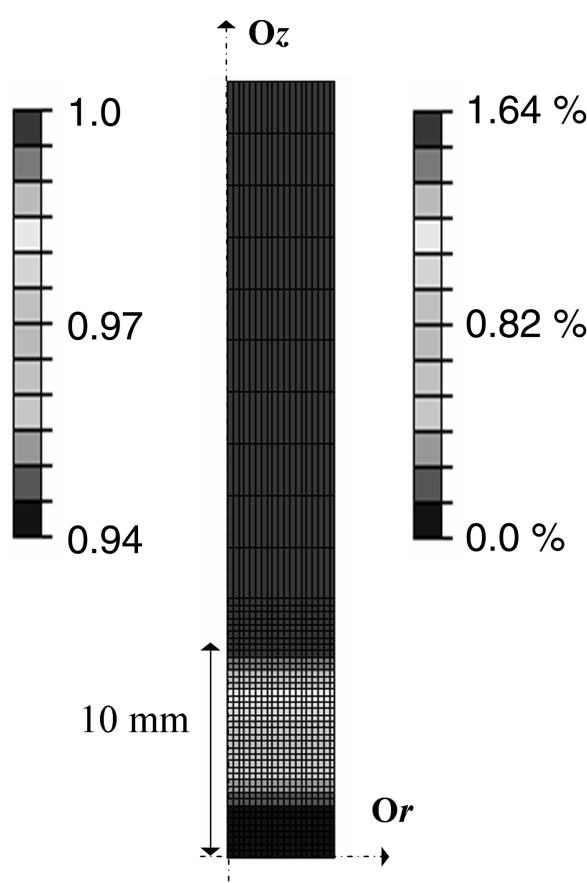

(a)

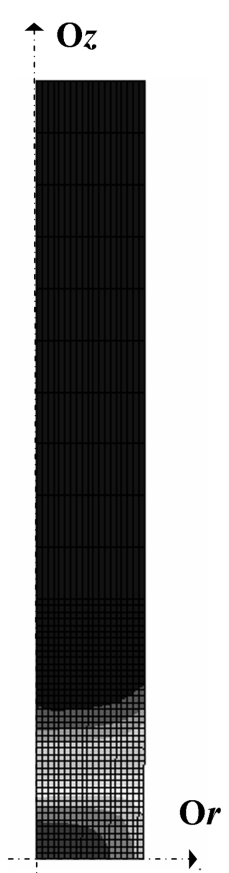

(b)
Fig. 12-(a) Solid fraction distribution calculated by Abaqus for a solid fraction equal to 0.94 in the middle of the tensile specimen. $(b)$ Axial strain distribution calculated for the value of macroscopic displacement that corresponds to fracture. Due to symmetries, only a quarter of the longitudinal section of the specimen is represented.

axisymmetric conditions were used. The axial displacement was imposed on the upper boundary of the computation domain and the sum of the reaction forces was recorded in order to estimate the resistance of the specimen to the deformation. The temperature distribution (and associated solid fraction distribution) was determined by thermal measurements carried out on nondeformed test samples. The mesh was refined close to the center of the specimen where most of the deformation takes place owing to the solid fraction gradient. On the other hand, it was rather coarse in the fully 
solid region. Linear axisymmetric elements were used and the constitutive model presented in Section II was implemented in the user routine CREEP. The goal of these numerical simulations was to compute not only the stress-displacement curves shown in Figure 9 but also to estimate the strain encountered in the mushy zone at the onset of fracture (c.f. Figure 12(b)).

For the shear and drained compression tests, elasticity has been neglected since large plastic strains were at play. In tension, the small displacements that characterize the fracture behavior may indicate that elasticity plays a role in the measured stress-displacement curves. Very little is known concerning the elastic properties of partially solidified alloys. We used the experimental data of Decultieux, ${ }^{[36]}$ who has collected unloading portions of simple compression tests on reheated Al-Cu specimens to evaluate the Young's modulus. For solid fractions that are of interest here, his data suggest that $E=10 \mathrm{GPa}$ is a reasonable approximation. This value is in good agreement with Grindosonic measurements carried out at very high homologous temperature on an $\mathrm{Al}-\mathrm{Cu}$ alloy. ${ }^{[33]}$

We propose simple expressions for the rheological functions $C^{*}$ and $\alpha$ that are valid in tensile conditions. We assume that the saturation stress that corresponds to the $C=$ $C^{*}$ condition cannot be attained in tension because fracture occurs much earlier. This is realistic since we have shown that the saturation value of $C^{*}$ is reached for large strains (Figure 4) that are not compatible with fracture strains observed in tension. Thus, we simply state that the saturation value of $C$ for $X<0$ is the same as in pure shear:

$$
C^{*}\left(g_{s}, X<0\right)=C^{*}\left(g_{s}, 0\right)=1-\left(1-g_{s}\right)^{p}
$$

The comparison of the experimental results in shear (Figure 4) and in tension (Figures 9 and 10) shows that the mechanical effect of coalescence could not be detected in pure shear, whereas it affects significantly the response of the solidifying alloy in tension. We believe that the solid bridges evidenced in Figure 11 act as the main obstacle to fracture in tension. Conversely, after the gradual fracture of these solid bridges in pure shear, flow resistance can still develop due to dendrite interactions (entanglement). For more compressive stress states (drained compression), the effect of solid bridges brought by coalescence should be negligible since densification of the solid skeleton brings many new contacts between dendrites. Thus, the effect of coalescence should be taken into account only for tensile stress states and should act at small strains for which solid bridges have not fractured yet. This is done by introducing the coalescence solid fraction $g_{s}^{\text {coal }}$ in the $\alpha$ function for tensile conditions:

$$
\alpha\left(g_{s}, X<0\right)=\alpha_{0}+\alpha_{1} \frac{g_{s}^{\frac{1}{3}}}{1-g_{s}^{\frac{1}{3}}} \exp \left(k\left(g_{s}-g_{s}^{\text {coal }}\right)\right)
$$

where $k$ is a parameter that accounts for the effect of coalescence on the tensile behavior. Equation [21] is very similar to the expression proposed for pure shear (Eq. [15]). We have found that a value of $k$ of 100 is necessary to account for the abrupt change of behavior in tension for solid frac- tions larger than $g_{s}^{\text {coal }}$. Note that for $g_{s}<g_{s}^{\text {coal }}$, the $\alpha$ function is approximately constant $\left(\alpha \approx \alpha_{0}\right)$. The results of the finite-element simulations are shown in Figure 9 (dotted curves). The comparison with experimental data is far from perfect, especially at very high solid fraction. However, it should be noticed that in comparing experimental and calculated stress-displacement curves, we are examining a very sensitive measure of the predictive capability of the model. This is due to the very small strains that are at play for this stress state and to the severe solid fraction and strain gradients that characterize the tensile test (Figures 12(a) and (b)). In that context, we believe that the simple function given in Eq. [21] associated with the flow equation proposed in Section II is able to reproduce fairly well the experimental data.

As previously stated, the model, in its present form, is not intended to replicate the details of the fracture phenomena (especially the abrupt softening after the maximum stress). However, we believe that it can still give valuable information concerning, for example, the critical strain that characterizes fracture by comparing the experimental curves to the calculated ones. Figure 13 shows the evolution of the axial strain in the central element calculated at the displacement that is associated to fracture in the corresponding experimental curve. Note that we have not observed a relation between the refinement of the finiteelement mesh and the value of this critical strain. The main observation from Figure 13 is that there is a large scatter in the strain that corresponds to fracture of the specimen, even more pronounced at high solid fraction. This is to be expected since we are dealing with a fracture phenomenon. In any case, Figure 13 shows that strain at fracture is of the order of a few percent confirming the fragile behavior of the mush when strained above the solidus temperature.

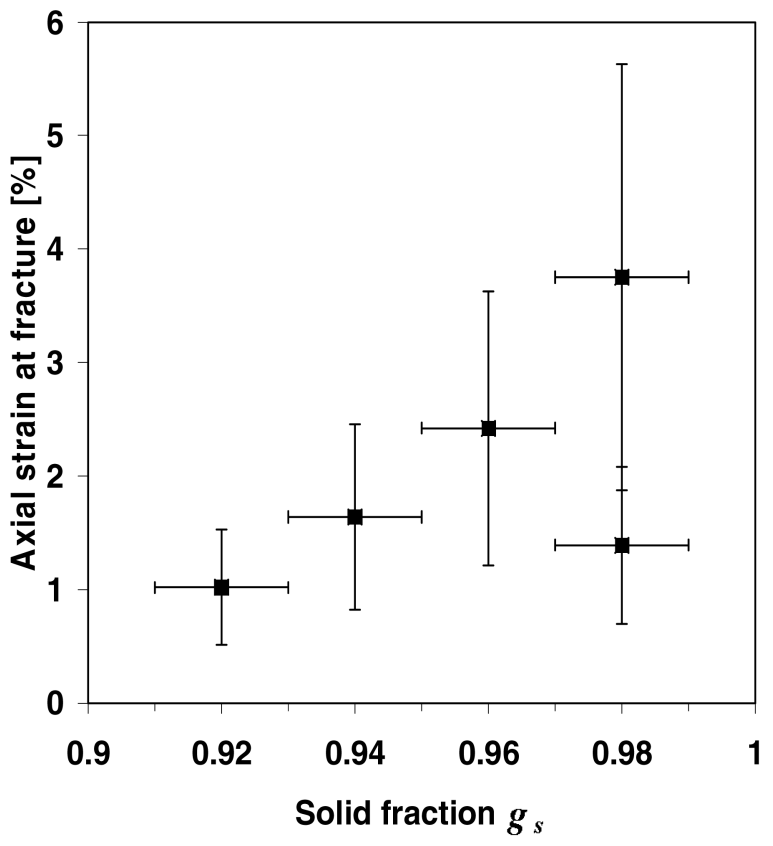

Fig. 13-Axial strain in the central element calculated for a value of the displacement corresponding to the fracture of the specimen, plotted as a function of the tested solid fraction. 


\section{CONCLUDING REMARKS}

The rheology of a solidifying alloy has been modeled by introducing an internal variable that represents the partial cohesion of the mush into the constitutive equation of a viscoplastic porous medium saturated with liquid. Although the model has been identified with only three points in the stress space, it is capable of predicting the stress-strain response of the solidifying alloy in the entire stress space. In a phenomenological framework, the accuracy of the prediction will depend on the number of tests used in the stress space to identify the model parameters. The development of the model equations has been directed toward the prediction of the rheological behavior of a solidifying alloy in tension or close to tensile stress states. In that context, we believe that the three stress states that have been used here (pure shear, drained compression, and simple tension) should be sufficient to provide some confidence when using the model parameters.

The functions that appear in the model have been intentionally kept as simple as possible to ease the implementation of the model in a finite-element code. The isopotential curves (Eq. [12]) are simple elliptic curves in the stress space that are available in most commercial codes. The model proposed here departs from standard models for viscoplastic porous media because it introduces an internal variable that accounts for the partial cohesion of the mush. In particular, the strong dissymmetry between tensile and compressive stress states is rendered through the evolution of the internal variable $C$. The two functions $\alpha$ and $C^{*}$ that dictate the evolution of $C$ introduce critical solid fractions that may be linked to microstructural features. The coherency solid fraction, $g_{s}^{\mathrm{coh}}$, should depend strongly on the morphology of the solid grains with inoculated alloys likely to exhibit higher value of $g_{s}^{\text {coh }}$ due to the more rounded morphology of the grains. The value of the coalescence solid fraction, $g_{s}^{\text {coal }}$, will define the stage of the solidification process at which tensile stresses start to build up sharply in the model. The coalescence solid fraction, $g_{s}^{\text {coal }}$, is related to the morphology of grains but more significantly to the interfacial energies between solid and liquid phases. ${ }^{[13]}$ Above this solid fraction, the mush starts to approach the behavior of a fully cohesive porous medium. Clearly, this critical solid fraction is of great importance to model the occurrence of hot tearing. This is because stresses may build up considerably around this solid fraction with the volume element (or its close surroundings) still lacking ductility. By introducing explicitly this critical solid fraction, the model offers the possibility to track the effects of accelerating or retarding coalescence on hot tearing. However, it should be clear that the $g_{s}^{\text {coh }}$ and $g_{s}^{\text {coal }}$ values are valid only for a grain-refined structure, as studied experimentally here.

Although the dilatation of the solid skeleton is present in the model framework when tensile strains are at play, we have not observed any significant softening effect associated with it in the simulations. This is because the tensile strains that are at play are too small to cause significant softening. In other words, the solidifying alloy exhibits a fragile behavior and gradual damage does not take place at a scale that can be captured by the model. However, the capacity of the model to reproduce the possibility of the mush to densify (by expelling liquid) or to dilate (by draining liquid) may prove useful to simulate macrosegregation phenomena that are encountered in solidification processes.

\section{ACKNOWLEDGMENTS}

This research was carried out as part of the Fifth Framework Competitive and Sustainable Growth Programme Project No. GRD1-1999-10921 VIRCAST (Contract No. G5RD-CT-2000-00153). This project included the following partners as major subcontractors: Calcom SA (Lausanne, Switzerland), Elkem Aluminum ANS (Norway), École Polytechnique Fédérale de Lausanne (Switzerland), CORUS Technology BV (The Netherlands), Hydro Aluminum AS (Norway), Institut National Polytechnique de Grenoble (Grenoble, France), Institut National Polytechnique de Lorraine (France), Norwegian University of Science and Technology (Norway), Alcan France (formerly Pechiney SA) (France), Hydro Aluminium Deutschland GmbH (Germany), and IFE and SINTEF (Norway). Funding by the European Community and by the Office Fédéral de l'Education et de la Science (Bern) for the Swiss partners is gratefully acknowledged.

\section{APPENDIX}

Summary of the Model Functions and Rheological Parameters

We summarize the functions that appear in the evolution Eq. [14]:

$$
\begin{gathered}
C^{*}\left(g_{s}, X \leq 2\right)=1-\left(1-g_{s}\right)^{p} \\
C^{*}\left(g_{s}, X>2\right)=1 \\
C^{*}\left(g_{s} \leq g_{s}^{\mathrm{coh}}, X\right)=0
\end{gathered}
$$

Note that a continuous increase of $C^{*}$ may be used instead of the two expressions proposed previously when the triaxiality $X$ increases. For the stress states studied here, we have verified that this does not modify significantly our simulation results.

$$
\begin{gathered}
\alpha\left(g_{s}, X<0\right)=\alpha_{0}+\alpha_{1} \frac{g_{s}^{\frac{1}{3}}}{1-g_{s}^{\frac{1}{3}}} \exp \left(k\left(g_{s}-g_{s}^{\text {coal }}\right)\right) \\
\alpha\left(g_{s}, X \geq 0\right)=\alpha_{0}+\alpha_{1} \frac{g_{s}^{\frac{1}{3}}}{1-g_{s}^{\frac{1}{3}}} \\
\alpha\left(g_{s} \leq g_{s}^{\text {coh }}, X\right)=0
\end{gathered}
$$

Again, a continuous expression of $\alpha$ with the triaxiality $X$ may be used as an alternative to the two expressions

Table A1. Rheological Parameters Used in the Model

\begin{tabular}{lccccccccc}
\hline$p$ & $\alpha_{0}$ & $\alpha_{1}$ & $g_{s}^{\text {coh }}$ & $g_{s}^{\text {coal }}$ & $k$ & $n$ & $s_{0}(\mathrm{MPa})$ & $A\left(\mathrm{~s}^{-1}\right)$ & $Q(\mathrm{~kJ} / \mathrm{mol})$ \\
\hline 0.11 & 4.45 & $1.07 \times 10^{-2}$ & 0.65 & 0.94 & 100 & 3.8 & 4.77 & $9 \cdot 10^{5}$ & 154 \\
\hline
\end{tabular}


given previously without significant effects on simulation results.

\section{NOMENCLATURE}

A rheological parameter of the fully solid material $A_{2}, A_{3} \quad$ solid fraction functions

$C$ internal variable representing the partial cohesion of the mush

$C^{*}\left(g_{s}, X\right)$ rheological function (cohesion at saturation)

$E \quad$ Young's modulus

$F \quad$ softening function relating $\Omega^{0}$ to $\Omega^{1}$

$g_{s}, g_{s}^{0} \quad$ volumetric solid fraction, initial value in the drained test

$g_{s}^{\text {coh }}, g_{s}^{\text {coal }} \quad$ coherency solid fraction and coalescence solid fraction

$k \quad$ parameter of the $\alpha\left(g_{s}, X\right)$ function

$n \quad$ power-law coefficient

$p \quad$ parameter of the $C^{*}$ function

$p_{l} \quad$ interstitial liquid pressure

$\bar{P}_{s} \quad$ effective pressure on the solid phase (taken pos-

itive in compression)

$Q \quad$ activation energy

$s_{0} \quad$ average isotropic resistance to plastic flow for

the fully solid material

$\mathbf{S}_{s} \quad$ solid-phase deviatoric effective stress tensor

$T, \dot{T} \quad$ temperature and cooling rate

$X \quad$ stress triaxiality

\section{$\underline{\text { Greek letters }}$}

$\alpha\left(g_{s}, X\right) \quad$ rheological function with parameters $\alpha_{0}$ and $\alpha_{1}$ $\varepsilon_{e}^{a c c} \quad$ accumulated macroscopic strain (plastic)

$\dot{\varepsilon}_{0} \quad$ reference strain rate for the fully solid material

$\dot{\varepsilon}_{e} \quad$ scalar measure of the macroscopic plastic strain rate of the solid phase

$\dot{\varepsilon}_{s}^{p} \quad$ plastic strain rate tensor of the solid phase

$\Omega^{0}, \Omega^{1}, \Omega$ viscoplastic potential of the fully solid material, of the fully cohesive porous material, and of the partially cohesive porous material, respectively

$\sigma$

$\bar{\sigma}_{s}$

$\hat{\sigma}_{\mathrm{s}}$

von Mises stress of the solid phase

effective stress tensor on the solid phase

\section{REFERENCES}

1. A.K. Dahle and L. Arnberg: JOM, 1996, vol. 48, pp. 34-37.

2. A.K. Dahle and D.H. StJohn: Acta Mater., 1999, vol. 47, pp. 31-41.

3. A.K. Dahle, S. Instone, and T. Sumitomo: Metall. Mater. Trans. A, 2003, vol. 34A, pp. 105-13.

4. I. Farup and A. Mo: Metall. Mater. Trans. A, 2000, vol. 31A, pp. 1361-472.

5. M. M'hamdi, A. Mo, and C.L. Martin: Metall. Mater. Trans. A, 2002, vol. 33A, pp. 2081-93.

6. S. Le Corre, M. Bellet, F. Bay, and Y. Chastel: in Modelling of Casting, Welding, and Advanced Solidification Processes X, D.M. Stefanescu,

J. Warren, M. Jolly, and M. Krane, eds., TMS, Warrendale, PA, 2003, pp. $345-52$.

7. M. Rappaz, J.-M. Drezet, and M. Gremaud: Metall. Mater. Trans. A, 1999, vol. 30A, pp. 449-56.

8. C.L. Martin, O. Ludwig, and M. Suéry: World Congr. on Computational Mechanics V (WCCM V), H.A. Mang; F.G. Rammerstorfer, and J. Eberhardsteiner, eds., Vienna University of Technology, Vienna, 2002 (CD Rom).

9. C.L. Martin, M. Braccini, and M. Suéry: Mater. Sci. Eng. A, 2002, vol. A325, pp. 293-302.

10. O. Ludwig, C.L. Martin, and M. Suéry: Int. Conf. on Aluminium Alloys 8, P.J. Gregson and S.J. Harris, eds., Cambridge, United Kingdom; Mater. Sci. Forum, 2002, vols. 396-402, pp. 265-70.

11. O. Ludwig, B. Commet, J.-M. Drezet, C.L. Martin, and M. Suéry: in Modeling of Casting, Welding and Advanced Solidification Processes $X$, Stefanescu-Waren-Jolly-Krane, eds., TMS, Warrendale, PA, 2003, pp. $183-90$.

12. J.-M. Drezet, O. Ludwig, M. M'hamdi, H.G. Fjaer, and C.L. Martin: in Light Metals 2004, T. Tabereaux, eds., TMS, Warrendale, PA, 2004, pp. $655-60$

13. V. Mathier, A. Jacot, and M. Rappaz: Modelling Simul. Mater. Eng., 2004, vol. 12, pp. 479-90.

14. C.L. Martin, D. Favier, and M. Suéry: Int. J. Plasticity, 1997, vol. 13 (3), pp. 215-35.

15. C.L. Martin, D. Favier, and M. Suéry: Int. J. Plasticity, 1997, vol. 13 (3), pp. 237-59.

16. C.L. Martin, D. Favier, and M. Suéry: Int. J. Plasticity, 1999, vol. 15, pp. 981-1008.

17. A. Zavaliangos: Int. J. Mech. Sci., 1998, vol. 40, pp. 1029-41.

18. A. Zavaliangos and L. Anand: J. Mech. Phys. Solids, 1993, vol. 41, pp. 1087-18.

19. O. Ludwig: Ph.D. Thesis, Institut National Polytechnique de Grenoble, Grenoble, 2004.

20. J.C. Michel and P. Suquet: J. Mech. Phys. Solids, 1992, vol. 40, pp. 783-812.

21. J.M. Duva and P.D. Crow: Acta Metall., 1992, vol. 40, pp. 31-35.

22. C.L. Martin, P. Kumar, and S.B. Brown: Acta Metall. Mater., 1994, vol. 42 , pp. 3603-14.

23. C. Sigli, L. Maenner, C. Sztur, and R. Shahani: Proc. Int. Conf. on Aluminum Alloys, T. Sato, S. Kumai, T. Kobayashi, and Y. Murakami, eds., JILM, Tokyo, 1998, pp. 87-98.

24. M. Braccini, C.L. Martin, A. Tourabi, Y. Bréchet, and M. Suéry: Mater. Sci. Eng. A, 2002, vol. A337, pp. 1-11.

25. N. Cristescu: Int. J. Plasticity, 1994, vol. 10, pp. 103-31.

26. W.S. Pellini: Strain Theory of Hot Tearing, Foundry, 1952, pp. 125-99.

27. G.K. Sigworth: AFS Trans., 1996, vol. 106, pp. 1053-59.

28. T.W. Clyne and G.J. Davies: Solidification Casting Met., Metals Society, London, 1979, pp. 275-78.

29. T.W. Clyne and G.J. Davies: Br. Foundryman, 1981, vol. 74, pp. 65-73.

30. B. Magnin, L. Maenner, L. Katgerman, and S. Engler: Mater. Sci. Forum, 1996, vols. 217-222, pp. 1209-14.

31. D.J. Lahaie and M. Bouchard: Metall. Mater. Trans. B, 2001, vol. 32B, pp. 697-705.

32. D.G. Eskin, Suyitno, and L. Katgerman: Progr. Mater. Sci., 2004, vol. 49, pp. 629-711.

33. M. Suéry, Y. Bréchet, M. Braccini, and C.L. Martin: Adv. Eng. Mater., 2001, vol. 3, pp. 589-93.

34. M. Rappaz, A. Jacot, and W.J. Boettinger: Metall. Mater. Trans. A, 2003, vol. 34A, pp. 467-79.

35. M. Rappaz, J.-M. Drezet, P.-D. Grasso, and A. Jacot: in Modeling of Casting, Welding and Advanced Solidification Processes X, Stefanescu-Waren-Jolly-Krane, eds., TMS, Warrendale, PA, 2003, pp. $53-60$

36. F. Decultieux: Ph.D. Thesis, Ecole des Mines de Paris, Paris, 1996. 